\title{
Zur Kinetik der strahleninduzierten Wasserstoffabspaltung aus Thymin
}

\author{
D. Heitkamp, O. Merwitz und H. Späth * \\ Kernforschungszentrum Karlsruhe, Institut für Radiochemie
}

(Z. Naturforsch. 23 b, 403-412 [1968] ; eingegangen am 7. Juli 1967)

\begin{abstract}
Im Anschluß an Messungen der Tritiumabspaltung aus $\gamma$-bestrahltem Thymin-(methyl-T) und Thymin-6-T wird ein kinetisches Modell für die während der Bestrahlung ablaufenden Reaktionen diskutiert. Die auf Grund dieses Modells berechneten Dosiseffektkurven stimmen mit den gemessenen im Dosisbereich $10^{5}-5 \cdot 10^{8} \mathrm{rad}$ sehr gut überein.
\end{abstract}

Die Vorgänge, die sich bei der Bestrahlung chemischer oder biologischer Objekte abspielen, sind im allgemeinen außerordentlich komplex. Man unterscheidet nach Platzman 1, 2 drei zeitlich aufeinanderfolgende und teilweise sich überschneidende Phasen im Mechanismus der Strahlenschädiung. In der physikalischen Phase wird kurzwellige Strahlung absorbiert, wobei hochangeregte Moleküle entstehen, die ihrerseits prädissoziieren, dissoziieren oder ionisiert werden können. Die dabei entstehenden sogenannten Primärprodukte der Bestrahlung, wie angeregte, dissoziierte, ionisierte Moleküle oder freie Elektronen, sind meist äußerst kurzlebig und instabil und reagieren in der zweiten oder physikochemischen Phase zu etwas langlebigeren Zwischenprodukten (freie Radikale), die in die eigentliche chemische Phase eintreten und dort zu den experimentell gefundenen Endprodukten permanenten Strahlenschadens weiterreagieren. Da theoretisch eine Vielzahl von möglichen Zwischenprodukten denkbar ist, die wieder in verschiedener Weise weiterreagieren können, ist es sehr schwierig, den Mechanismus in der zweiten und dritten Phase aufzuklären und so zu einer quantitativen Deutung der Dosiseffektkurven zu kommen.

Die meisten in der Literatur bekannten Analysen sind treffertheoretischer Natur. Derartige Aussagen liefern zwar eine mathematische Beschreibung der experimentell gefundenen Dosiseffektkurven, lassen jedoch im allgemeinen keine eindeutige Entscheidung über die Natur des Trefferereignisses und die Trefferzahl zu ${ }^{3,4}$, vor allem dann nicht, wenn Erholungsvorgänge eine wesentliche Rolle spielen. In jüngster Zeit haben daher verschiedene Autoren begonnen, auf reaktionskinetischer Basis zu einer quantitativen Deutung der experimentellen Dosiseffektivkurven zu

\footnotetext{
* Institut für Neutronenphysik und Reaktortechnik.

1 R. L. Platzman, Radiat. Res. 17, 419 [1962].

2 R. L. Platzman, The Vortex 23, 372 [1962].
}

gelangen. Kellerer und Hug ${ }^{3,4}$ diskutieren Alternativen zu den trefferthoretischen Ansätzen und liefern mit stochastischen Methoden ein mathematisches Schema, das eine verallgemeinerte Darstellung der Treffertheorie unter Berücksichtigung von Erholungsvorgängen ebenso gestattet wie die Darstellung strahleninduzierter Reaktionsabläufe auch komplexer Systeme auf kinetischer Grundlage. In ähnlicher Weise behandelt Dienes ${ }^{5}$ die mathematischen Grundlagen einer linearen Reaktionskette, d. h. einer Folge von Reaktionen 0. und 1. Ordnung, ihre Beziehung zu treffertheoretischen Aussagen und ihre Anwendung auf Überlebensraten bestrahlter biologischer Objekte. Dabei werden jedoch keine Angaben über die Natur der einzelnen Reaktionsschritte gemacht außer, daß sie niederer Ordnung sind. Die Kenntnis dieser Reaktionen ist natürlich für das detaillierte Verständnis des Mechanismus der chemischen Phase einer Strahlenschädigung von großem Interesse. Die Grundlagen dazu sind in letzter Zeit durch die erfolgreiche Anwendung der ESR-Spektroskopie geschaffen worden, mit deren Hilfe man die Existenz verschiedener Radikale als Zwischenprodukte im Reaktionsmechanismus nachweisen oder zumindest wahrscheinlich machen konnte.

Davon ausgehend soll in dieser Arbeit der Versuch gemacht werden, im Falle der Wasserstoffabspaltung aus der DNS-Komponente Thymin ein spezifisches kinetisches Modell anzugeben, das die gemessenen Dosiseffektkurven quantitativ zu deuten gestattet und gegebenenfalls Rückschlüsse auf die relative Bedeutung der einzelnen dem Modell zugrundeliegenden Reaktionen in Abhängigkeit von der Dosis erlaubt.

\footnotetext{
3 A. Kellerer u. O. Hug, Biophysik 1, 20 [1963].

4 A. Kellerer u. O. Hug, Biophysik 1, 33 [1963].

5 G. J. Dienes, Radiat. Res. 28, 183 [1966].
} 


\section{Experimentelle Ergebnisse}

Kürzlich hat Merwitz ${ }^{6}$ die Dosiseffektkurven für die Wasserstoffabspaltung aus $\gamma$-bestrahltem ${ }^{3} \mathrm{H}$-Thymin im Dosisbereich von $10^{5}$ bis $5 \cdot 10^{8} \mathrm{rad}$ bestimmt. Die drei positionsmarkierten Thymine [Thy. min-(methyl-T), Thymin-6-T und Thymin-1,3-T] wurden im festen Zustand unter Vakuum der Bestrahlung mit einer ${ }^{60} \mathrm{Co}$-Gammaquelle (Genauigkeit der Dosiswerte $\pm 5 \%$ ) ausgesetzt. Der Strahlenschaden manifestierte sich bei Thymin-(methyl-T) und Thymin-6-T in der Bildung von Tritiumwasserstoff, bei Thymin-1,3-T in der Entstehung von Tritiumwasser. Die Aktivitätsbestimmung der festen tritiummarkierten Thymine erfolgte mittels Flüssigkeitsszintillation. Zur Messung der Aktivität in der Gasphase nach Bestrahlung und Sublimation des Thymins diente ein Gasfüllzählrohr. Die Eichung der Zählanordnung wurde durch Reduktion eines Tritiumwasserstandards mittels Zink zu Tritiumwasserstoff nach der Methode von Spernol und Denecke ${ }^{7}$ vorgenommen. Die Dosiseffektkurven der Tritiumwasserstoffabspaltung aus Thymin-(methyl-T) mit der spezifischen Aktivität $0,5 \mathrm{mCi} / \mathrm{g}$ und Thymin-6-T mit der spezifischen Aktivität $0,1 \mathrm{mCi} / \mathrm{g}$ sind in Abb. 2 im doppeltlogarithmischen Maßstab dargestellt; die entsprechenden Meßwerte sind in den Tabn. 1 und 2 aufgeführt. Der Gesamtfehler der Tritiumbestimmung beträgt maximal $\pm 5 \%$. Die Aktivität des Tritiumwasserstoffs in Zerfällen/min' $g$ ist der Gesamtmenge des abgespaltenen Wasserstoffs proportional.

Die Tritiumwasserabspaltung aus Thymin-1.3-T führte zu einer Kontamination des Zählrohrs und verhinderte dadurch reproduzierbare Messungen.

\section{Zur Kinetik der Wasserstoffbildung}

Bei der Aufstellung eines möglichen Reaktionsschemas, das zur Bildung molekularen Wasserstoffs als Folge der Bestrahlung führt, hat man zunächst alle theoretischen Möglichkeiten von Reaktionsteilschritten in Betracht zu ziehen ${ }^{6}$. Nach Absorption eines Gammaquants entstehen zunächst hochangeregte Thyminmoleküle$$
\mathrm{MH} \rightarrow \mathrm{MH}^{*}
$$$$
\mathrm{MH}=\text { Thymin (Abb. 1). }
$$

6 O. Merwitz, KFK-590 [1967].

7 A. Spernol u. B. Denecke, Int. J. Appl. Rad. Isot. 15, 139 [1964].

8 R. BraAms, Nature [London] 200, 752 [1963].
}

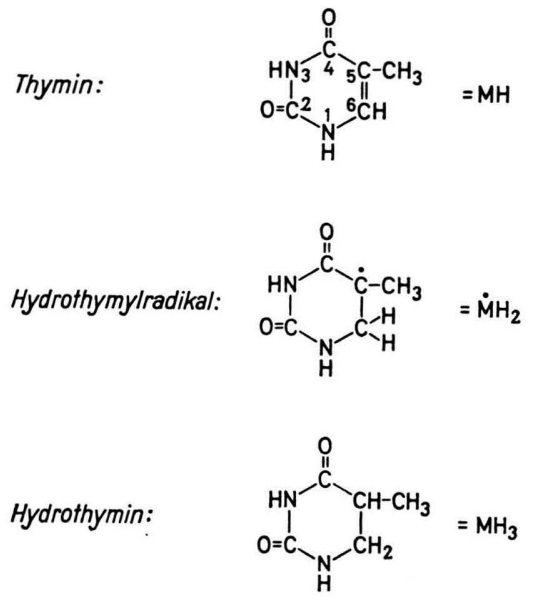

Abb. 1. Bedeutung der im Text verwandten Symbole.

Platzman $^{2}$ hat darauf hingewiesen, daß den angeregten Zuständen in der Strahlenchemie eine große Bedeutung zukommt. Ein bestimmter Bruchteil von $\mathrm{MH}^{*}$ wird in diesem Zustand verbleiben. Man muß daher annehmen, daß hochangeregte Thyminmoleküle stets in endlicher Konzentration während der Bestrahlung vorliegen.

Nach einer Hypothese von BraAms ${ }^{8}$ spielen vor allem H-Atome bei der Strahlenschädigung organischer Moleküle eine bedeutsame Rolle. Tatsächlich haben Patten und Gordy 9 in wäßriger Nucleinsäure bei der Temperatur des flüssigen Heliums $\left(4,2{ }^{\circ} \mathrm{K}\right)$ durch ESR-Spektroskopie H-Atome gefunden. Müller und Dertinger ${ }^{10}$ konnten neuerdings in trockener Bakteriophagen-DNS bei $77^{\circ} \mathrm{K}$ atomaren Wasserstoff spektroskopisch nachweisen, der bis zu Temperaturen von $200{ }^{\circ} \mathrm{K}$ meßbar bleibt. Bei der Bestrahlungstemperatur in der von Merwitz benutzten Kobaltquelle (ca. $50{ }^{\circ} \mathrm{C}$ ) sind demnach auch $\mathrm{H}$-Atome als Reaktionsträger anzunehmen, jedoch in derart geringer Konzentration, daß sie für einen direkten experimentellen Nachweis nicht ausreicht. In Analogie zur Hypothese von BraAms ${ }^{8}$ wird daher die Bildung atomaren Wasserstoffs als die die chemische Phase einleitende Reaktion angesehen:

$$
\mathrm{MH}^{*}=\mathrm{M}+\mathrm{H} \text {. }
$$

$\mathrm{M}=$ Thymylradikal.

Reaktion 1 ist als eine summarische Reaktion aufzufassen, die nicht nur unmittelbar in der angegebe-

9 R. A. Patten u. W. Gordy, Nature [London] 201, 361 [1964].

10 A. Müller u. H. Dertinger, Z. Naturforschg. 23 b, 83 [1968]. 
nen Weise, sondern über mehrere Teilschritte der physikalischen und physikochemischen Phase verläuft. Auf die Natur dieser Teilschritte soll hier nicht näher eingegangen werden. Die in der Literatur vorgeschlagenen Mechanismen sind noch weitgehend hypothetisch.

Die nach Reaktion 1 gebildeten Radikale können entweder spontan oder statistisch rekombinieren. Unter spontaner Rekombination soll in Analogie zu den Vorstellungen über die Strahlenschädigung fester Stoffe die Verbindung von M-Radikalen mit H-Atomen in ihrer unmittelbaren Umgebung verstanden werden. In dieser Umgebung, die im allgemeinen 50-100 Moleküle umfaßt, wird die Wahrscheinlichkeit einer Rekombination nur von der Summe (nicht vom Produkt) der vorhandenen Radikale abhängen. Es handelt sich also um eine Reaktion 1. Ordnung:

$$
\mathrm{M}+\mathrm{H} \stackrel{\mathrm{sp} .}{\rightarrow} \mathrm{MH}
$$

Im Gegensatz dazu ist die statistische Rekombination außerhalb dieses Bereichs (Reaktion 7) von 2. Ordnung. Ähnliche Überlegungen gelten auch für die Vereinigung von H-Atomen untereinander. Auch hier kann zwischen spontaner Rekombination in begrenzten Bereichen

$$
\mathrm{H}+\mathrm{H} \stackrel{\text { sp. }}{\rightarrow} \mathrm{H}_{2}
$$

und statistischer Rekombination (Reaktion 6) unterschieden werden.

Die nach Reaktionsgleichung 1 gebildeten $\mathrm{H}$ Atome, die als energiereich und sehr beweglich anzusehen sind, können außer der spontanen Rekombination verschiedene Folgereaktionen bewirken. 1959 entdeckten SHIElds und GoRdy ${ }^{11}$ mit Hilfe der ESRSpektroskopie das bei der Bestrahlung von DNS, Thymidin und Thymin auftretende langlebige $\mathrm{Hy}$ drothymylradikal $\mathrm{MH}_{2}$ (Abb. 1), dessen Entstehung später auf die Anlagerung strahlenerzeugter $\mathrm{H}$ Atome an die 6-Position des Thymins zurückgeführt wurde ${ }^{12-16}$.

$$
\mathrm{MH}+\mathrm{H}=\mathrm{MH}_{2} \text {. }
$$

11 H. Shields u. W. Gordy, Proc. nat. Acad. Sci. USA 45, 269 [1959].

12 P. Pershan, R. Shulman, B. Wyiuda u. J. Eisinger, Physics 1, 163 [1964].

13 B. Pruden, W. Snipes u. W. Gordy, Proc. nat. Acad. Sci. USA 53, 917 [1965].

14 J. N. Herax u. W. Gordy, Proc. nat. Acad. Sci. USA 54, 1287 [1965].
Neben der Anlagerung findet die zur Bildung von molekularem Wasserstoff führende Abstraktion von H-Atomen aus ungeschädigten Thyminmolekülen statt:

$$
\mathrm{MH}+\mathrm{H}=\mathrm{M}+\mathrm{H}_{2} .
$$

Diese Reaktion wird von BraAms ${ }^{8}$ als Hauptlieferant des molekularen Wasserstoffs angesehen. SomMERMAYER et al. ${ }^{17}$ haben mit Reaktion 5 die $\mathrm{H}_{2}$-Bildung bei der Bestrahlung von Valin erklärt. Den statistischen Rekombinationen

$$
\text { und } \quad \begin{aligned}
& \mathrm{H}+\mathrm{H} \rightarrow \mathrm{H}_{2} \\
& \mathrm{M}+\mathrm{H} \rightarrow \mathrm{MH}
\end{aligned}
$$

dürfte geringere Bedeutung zukommen, da die HAtome wegen ihrer Reaktionsfreudigkeit nur eine geringe Konzentration erreichen.

Grundsätzlich möglich ist auch, daß das gebildete Thymylradikal $M$ in unmittelbarer Nachbarschaft mit einem Thyminmolekül bzw. einem weiteren Radikal $\mathbf{M}$ unter Bildung eines Dimerisationsproduktes MM reagiert ${ }^{6,17}$ :

$$
\begin{aligned}
\mathrm{MH}+\mathrm{M} & =\mathrm{MM}+\mathrm{H}, \\
\mathrm{M}+\mathrm{M} & =\mathbf{M M},
\end{aligned}
$$

oder daß benachbarte Thyminmoleküle unter Bildung molekularen Wasserstoffs dimerisieren:

$$
2 \mathrm{MH} \rightarrow \mathrm{MM}+\mathrm{H}_{2},
$$

eine Reaktion, die von Dole et al. ${ }^{18}$ bei der Bestrahlung von Polyäthylen diskutiert wird. Die von MER${ }_{\text {wITZ }}{ }^{6}$ nach der Bestrahlung von Thymin gefundenen nicht sublimierbaren Anteile könnten von dimerisierten Molekülen MM herrühren. Es finden sich in der Literatur wenig Anhaltspunkte dafür, inwieweit der bereits gebildete molekulare Wasserstoff wieder weiterreagieren kann, sofern er noch nicht aus dem bestrahlten festen Thymin herausdiffundiert ist. Folgende Reaktionen könnten ablaufen:

$$
\begin{aligned}
\mathrm{M}+\mathrm{H}_{2} & =\mathrm{MH}+\mathrm{H}, \\
\mathrm{MH}_{2}+\mathrm{H}_{2} & =\mathrm{MH}_{3}+\mathrm{H}, \\
\mathrm{MH}+\mathrm{H}_{2} & =\mathrm{MH}_{2}+\mathrm{H}, \\
\mathrm{MH}+\mathrm{H}_{2} & =\mathrm{MH}_{3} .
\end{aligned}
$$

$\mathrm{MH}_{3}=$ Hydrothymin (Abb. 1).

15 H. C. Heller u. T. Cole, Proc. nat. Acad. Sci. USA 54, 1486 [1965].

16 D. E. Holmes, L. S. Mrers u. R. B. Ingalls, Nature [London] 219, 1017 [1966].

17 K. Sommermeyer, J. Stegle u. G. H. Schnepel, Atompraxis 13, 20 [1967].

18 M. Dole, D. C. William u. A. J. Arvia, Proc. Int. Conf. Peaceful Uses of Atomic Energy, Paper 818 [1958]. 
Es ist zwar nicht anzunehmen, daß diese Reaktionen unter thermischen Bedingungen ablaufen, wohl aber, wenn eines der beiden reagierenden Moleküle sich in einem angeregten Zustand befindet. Hierbei wird es sich eher um nach Reaktion 1 gebildete hochanregte Thyminmoleküle $\mathrm{MH}^{*}$ handeln als um hochangeregte $\mathrm{H}_{2}$-Moleküle, die stärker zur Dissoziation neigen.

An dieser Stelle soll noch vermerkt werden, daß Hydrothymin statt durch Reaktion 13 auch durch Addition von $\mathrm{H}$-Atomen an Hydrothymylradikale entstehen kann:

$$
\mathrm{MH}_{2}+\mathrm{H}=\mathrm{MH}_{3} \text {. }
$$

\section{Das Reaktionsmodell}

Bei der Formulierung eines mathematisch einfachen kinetischen Modells für die $\mathrm{H}_{2}$-Abspaltung aus Thymin sollen zunächst nur Reaktionen 0 . und 1 . Ordnung berücksichtigt werden. Das erscheint gerechtfertigt, da Reaktionen 2. Ordnung im allgemeinen nur bei hohen Radikalkonzentrationen größere Bedeutung haben. Es entfallen damit die Reaktionen $6,7,9,11,12$ und 15. Ihr Einfluß soll später diskutiert werden. Desgleichen soll vorerst die Reaktion 8 unberücksichtigt bleiben. Von den beiden $\mathrm{H}_{2}$-verbrauchenden Reaktionen 13 und 14 erscheint Reaktion 13 weniger wahrscheinlich, da sie zur gleichzeitigen Bildung zweier Radikale führt. Sie wird im Anhang mit in Betracht gezogen.

Für die mathematische Analyse verbleiben daher folgende Reaktionen:

$$
\begin{aligned}
& \mathrm{MH}-\mathrm{MH}^{*} \quad \text { Anregung } \\
& \mathrm{MH}^{*} \stackrel{k_{1}}{\rightarrow} \mathrm{M}+\mathrm{H} \quad \text { Radikalbildung } \\
& \mathrm{M}+\mathrm{H} \stackrel{k_{2}}{\longrightarrow} \mathrm{MH} \quad \text { Spontane } \\
& \mathrm{H}+\mathrm{H} \stackrel{k_{3}}{\longrightarrow} \mathrm{H}_{2} \quad \text { Rekombination } \\
& \mathrm{MH}+\mathrm{H} \stackrel{k_{4}}{\rightarrow} \mathrm{MH}_{2} \quad \text { Anlagerung } \\
& \mathrm{MH}+\mathrm{H} \stackrel{k_{5}}{\longrightarrow} \mathrm{M}+\mathrm{H}_{2} \text { Abstraktion } \\
& 2 \mathrm{MH} \stackrel{k_{10}}{\longrightarrow} \mathrm{MM}+\mathrm{H}_{2} \text { Dimerisierung } \\
& \mathrm{MH}+\mathrm{H}_{2} \stackrel{k_{14}}{\rightarrow} \mathrm{MH}_{3} \quad \mathrm{H}_{2} \text {-Verbrauch }
\end{aligned}
$$

$k_{\mathrm{i}}=$ Geschwindigkeitskonstanten.

Diese Reaktionen sind 0. bzw. 1. Ordnung, weil die Konzentration des Thymins während der Bestrahlung maximal innerhalb weniger Prozent als konstant angesehen werden kann. Bei konstanter Dosisleistung kann in erster Näherung auch die Konzentration von $\mathrm{MH}^{*}$ als konstant betrachtet werden.
Von der durch die Reaktionen 3, 5 und 10 gebildeten Gesamtmenge molekularen Wasserstoffs $\left[\mathrm{H}_{2}\right]$ kann nur ein bestimmter Bruchteil $\left[H_{2}\right]_{\mathrm{r}}$ mit angeregten Thyminmolekülen gemäß Reaktion 14 reagieren. Der Restwasserstoff $\left[\mathrm{H}_{2}\right]_{\mathrm{d}}$ diffundiert teilweise aus dem bestrahlten Festkörper in die umgebende Gasatmosphäre:

$$
\left[H_{2}\right]=\left[H_{2}\right]_{\mathrm{r}}+\left[H_{2}\right]_{\mathrm{d}} .
$$

Der Anteil $\left[H_{2}\right]_{\mathrm{d}}$ wird mit zunehmender Bestrahlungsdauer größer. Bei hoher Dosis ist

$$
\left[H_{2}\right]_{\mathrm{d}} \cong\left[H_{2}\right] .
$$

Da die Anteile $\left[H_{2}\right]_{\mathrm{r}}$ und $\left[\mathrm{H}_{2}\right]_{\mathrm{d}}$ an der Gesamtkonzentration $\left[\mathrm{H}_{2}\right]$ unbekannt sind, müssen sie getrennt behandelt werden, d. h., die Reaktionen 3, 5 und 10 müssen formal in zwei Teilreaktionen aufgespalten werden, die je zur Bildung von $H_{2 \mathrm{r}}$ bzw. $H_{2 \mathrm{~d}}$ führen:

$$
\begin{gathered}
\mathrm{H}+\mathrm{H} \stackrel{k_{3 \mathrm{r}}}{\rightarrow} \mathrm{H}_{2 \mathrm{r}} \\
\mathrm{H}+\mathrm{H} \stackrel{k_{3 \mathrm{~d}}}{\rightarrow} \mathrm{H}_{2 \mathrm{~d}} \\
\mathrm{MH}+\mathrm{H} \stackrel{k_{5 \mathrm{r}}}{\rightarrow} \mathrm{M}+\mathrm{H}_{2 \mathrm{r}} \\
\mathrm{MH}+\mathrm{H} \stackrel{k_{5 \mathrm{~d}}}{\rightarrow} \mathrm{M}+\mathrm{H}_{2 \mathrm{~d}} \\
2 \mathrm{MH}^{*} \stackrel{k_{10 \mathrm{r}}}{\longrightarrow} \mathrm{MM}+\mathrm{H}_{2 \mathrm{r}} \\
2 \mathrm{MH}^{*} \stackrel{k_{10 \mathrm{~d}}}{\rightarrow} \mathrm{MM}+\mathrm{H}_{2 \mathrm{~d}} .
\end{gathered}
$$

Für die Differentialquotienten der Konzentrationen des atomaren und des molekularen Wasserstoffs gilt in diesem Modell

$$
\begin{aligned}
& \frac{\mathrm{d}[H]}{\mathrm{d} D}= k_{1}[1-\varrho([M]+[H])] \\
&-2 k_{3}[H]-k_{4}[H]-k_{5}[H] \\
& \frac{\mathrm{d}\left[H_{2}\right]}{\mathrm{d} D}=k_{10}+k_{3}[H]+k_{5}[H]-k_{14}\left[H_{2}\right]_{\mathrm{r}}
\end{aligned}
$$

wobei $\varrho$ eine Maßzahl des Volumens ist, in dem spontane Rekombination stattfindet. Die Änderung von $\left[\mathrm{H}_{2}\right]_{\mathrm{d}}$ mit der Dosis wird durch die Reaktionen 3,5 und 10 bestimmt, die von $\left[H_{2}\right] \mathrm{r}$ zusätzlich durch die $\mathrm{H}_{2}$-verbrauchende Reaktion 14. Gl. (4) kann also aufgespalten werden in

$$
\frac{\mathrm{d}\left[H_{2}\right]_{\mathrm{r}}}{\mathrm{d} D}=k_{10 \mathrm{r}}+\left(k_{3 \mathrm{r}}+k_{5 \mathrm{r}}\right)[H]-k_{14}\left[H_{2}\right]_{\mathrm{r}}
$$

und

$$
\frac{\mathrm{d}\left[H_{2}\right]_{\mathrm{d}}}{\mathrm{d} D}=k_{10 \mathrm{~d}}+\left(k_{3 \mathrm{~d}}+k_{5 \mathrm{~d}}\right)[H] .
$$

Aus Gl. (1) folgt:

$$
\begin{aligned}
& k_{3}=k_{3 \mathrm{~d}}+k_{3 \mathrm{r}} \\
& k_{5}=k_{5 \mathrm{~d}}+k_{5 \mathrm{r}} \\
& k_{10}=k_{10 \mathrm{~d}}+k_{10 \mathrm{r}} .
\end{aligned}
$$


Nach den Experimenten von Pietsch ${ }^{19}$ ist es sinnvoll, für die M-Konzentration eine Exponentialfunktion in der Form

$$
[M]=\frac{k_{1}}{\sigma}[1-\exp (-\sigma D)]
$$

anzusetzen, die eine einfache Sättigungskurve mit der Steigerung $k_{1}$ im Nullpunkt beschreibt ( $\sigma=$ experimenteller Parameter). Mit dem Ansatz (8) läßt sich Differentialgleichung (3) mit der Anfangsbedingung $[H]=0$ für $D=0$ lösen. Es ergibt sich die Konzentration des atomaren Wasserstoffs zu

$$
\begin{aligned}
{[H]=\left[\frac{1}{\varkappa-\sigma}+\frac{\mathrm{k}_{1}-k_{2}{ }^{\prime}}{\varkappa}\right][1} & -\exp (-\varkappa D)] \\
& -\frac{1}{\varkappa-\sigma}[1-\exp (-\sigma D)]
\end{aligned}
$$

Darin bedeuten

$$
\varkappa=k_{1} \varrho+2 k_{3}+k_{4}+k_{5}
$$

und

$$
k_{2}^{\prime}=\frac{k_{1}^{2} \varrho}{\sigma} \text {. }
$$

Eliminiert man mit Hilfe von Gl. (9) die H-Konzentration aus Gl. (5), so ergibt sich eine Differentialgleichung für die $H_{2 \mathrm{r}}$-Konzentration:

$$
\begin{gathered}
\frac{\mathrm{d}\left[H_{2}\right]_{\mathrm{r}}}{\mathrm{d} D}=k_{10 \mathrm{r}}+\frac{1}{\varkappa}\left(k_{1}-k_{2}{ }^{\prime}\right)\left(k_{3 \mathrm{r}}+k_{5 \mathrm{r}}\right)[1-\exp (-\varkappa D)] \\
+\frac{k_{3 \mathrm{r}}+k_{5 \mathrm{r}}}{\varkappa-\sigma}[\exp (-\sigma D)-\exp (-\varkappa D)]-k_{14}\left[H_{2}\right]_{\mathrm{r}} .
\end{gathered}
$$

Setzt man die Lösung der Differentialgleichung (10) mit der Anfangsbedingung $\left[H_{2}\right]_{\mathrm{r}}=0$ für $D=0$ wie auch die H-Konzentration aus Gl. (9) in Gl. (4) ein, so ergibt sich nach Integration mit der Anfangsbedingung $\left[H_{2}\right]=0$ für $D=0$ folgende $H_{2}$-Konzentration:

$$
\begin{aligned}
{\left[H_{2}\right]=} & -f[1-\exp (-\varkappa D)]+g\left[1-\exp \left(-k_{14} D\right)\right]+ \\
& +h[1-\exp (-\sigma D)]+c D .
\end{aligned}
$$

Darin sind

$$
\begin{aligned}
f= & \frac{1}{\varkappa}\left(\frac{k_{1}-k_{2}^{\prime}}{\varkappa}+\frac{1}{\varkappa-\sigma}\right)\left(k_{3}+k_{5}-\frac{k_{14}\left(k_{a \mathrm{r}}+k_{5 \mathrm{r}}\right)}{k_{14}-\sigma}\right) \\
g= & \frac{\left(k_{1}-k_{2}{ }^{\prime}\right)\left(k_{3 \mathrm{r}}+k_{5 \mathrm{r}}\right)}{\varkappa}\left(\frac{1}{\varkappa-k_{14}}+\frac{1}{k_{14}}\right) \\
& +\frac{k_{3 \mathrm{r}}+k_{5 \mathrm{r}}}{\varkappa-\sigma}\left(\frac{1}{\varkappa-k_{14}}+\frac{1}{k_{14}-\sigma}\right)+\frac{k_{10 \mathrm{r}}}{k_{14}} \\
h= & \frac{1}{\sigma(\varkappa-\sigma)}\left(k_{3}+k_{5}-\frac{k_{14}\left(k_{3 \mathrm{r}}+k_{5 \mathrm{r}}\right)}{k_{14}-\sigma}\right) \\
\text { und } c= & \frac{\left(k_{1}-k_{2}{ }^{\prime}\right)\left(k_{3 \mathrm{~d}}+k_{5 \mathrm{~d}}\right)}{\varkappa}+k_{10 \mathrm{~d}} .
\end{aligned}
$$

19 E. Pietsch, Dissertation Universität Heidelberg u. KFK-513 [1967].
Für $\sigma<k_{14}<\varkappa$ sind alle Konstanten positive Größen. In diesem Fall hat die Funktion (11) einen Wendepunkt, der für

$$
h \sigma^{2} \exp \left(-\sigma D_{\mathrm{w}}\right) \ll g k_{14}^{2} \exp \left(-k_{14} D_{\mathrm{w}}\right)
$$

angenähert durch Gl. (16) wiedergegeben wird. Die zweite Exponentialfunktion in Gl. (11) wird durch die $\mathrm{H}_{2}$-verbrauchende Reaktion 14 bedingt, die letzte Exponentialfunktion durch die spontane Rekombination. Nimmt man entweder keinen unmittelbaren $\mathrm{H}_{2}$ Verbrauch oder keine spontane Rekombination an, so entfällt jeweils eine Exponentialfunktion. Ohne Reaktion 14 erhält man für $[H]$ wieder $\mathrm{Gl}$. (9) und für

$$
\begin{aligned}
{\left[H_{2}\right]=-f^{\prime}[1-\exp (-\varkappa D)] } & \\
& +h^{\prime}[1-\exp (-\sigma D)]+c^{\prime} D
\end{aligned}
$$

mit

$$
\begin{aligned}
& f^{\prime}=\frac{\left(k_{1}-k_{2}{ }^{\prime}\right)\left(k_{3}+k_{5}\right)}{\varkappa^{2}}+\frac{k_{3}+k_{5}}{\varkappa(\varkappa-\sigma)} \\
& h^{\prime}=\frac{k_{3}+k_{5}}{\sigma(\varkappa-\sigma)} \\
& c^{\prime}=k_{10}+\frac{\left(k_{1}-k_{2}^{\prime}\right)\left(k_{3}+k_{5}\right)}{\varkappa} .
\end{aligned}
$$

Für $\sigma<\varkappa$ sind $f^{\prime}$ und $h^{\prime}$ positiv und der Wendepunkt ist gegeben durch

$$
D_{\mathrm{w}}=\frac{\ln \frac{f^{\prime} x^{2}}{h^{\prime} \sigma^{2}}}{x-\sigma} .
$$

Läßt man die Reaktionen 2 und 3 außer acht, so ergeben sich folgende Konzentrationen des atomaren und des molekularen Wasserstoffs:

$$
\begin{gathered}
{[H]=\frac{k_{1}}{k_{4}+k_{5}}\left[1-\exp \left[-\left(k_{4}+k_{5}\right) D\right]\right]} \\
{\left[H_{2}\right]=-f^{\prime \prime}\left[1-\exp \left[-\left(k_{4}+k_{5}\right) D\right]\right]} \\
+g^{\prime}\left[1-\exp \left(-k_{14} D\right)\right]+c^{\prime \prime} D .
\end{gathered}
$$

Darin sind

$$
\begin{aligned}
& f^{\prime \prime}=\frac{k_{1}}{\left(k_{4}+k_{5}\right)^{2}}\left(k_{5}+\frac{k_{5} \mathrm{r} k_{14}}{k_{4}+k_{5}-k_{14}}\right) \\
& g^{\prime}=\frac{k_{10 \mathrm{r}}}{k_{14}}+\frac{k_{1} k_{5 \mathrm{r}}}{k_{14}\left(k_{4}+k_{5}-k_{14}\right)} \\
& c^{\prime \prime}=k_{10 \mathrm{~d}}+\frac{k_{1} k_{5 \mathrm{~d}}}{k_{4}+k_{5}} \text {. }
\end{aligned}
$$

Ist $k_{14}<\left(k_{4}+k_{5}\right)$, so sind $f^{\prime \prime}, g^{\prime}$ positiv, und der Wendepunkt liegt jetzt bei der Dosis

$$
D_{\mathrm{w}}=\frac{\ln \frac{f^{\prime \prime}\left(k_{4}+k_{5}\right)^{2}}{g^{\prime} k^{2}{ }_{14}}}{k_{4}+k_{5}-k_{14}} .
$$


Berücksichtigt man hier neben der $\mathrm{H}_{2}$-verbrauchenden Reaktion 14 der Vollständigkeit halber auch die Reaktion 13, so ändern sich in Gl. (15) nur die Werte der Konstanten, wie im Anhang näher ausgeführt ist.

Zur Klärung der Frage, inwieweit die auf der Basis des vorgeschlagenen Modells gefundene Abhängigkeit der $\mathrm{H}_{2}$-Konzentration von der Dosis die experimentelle Kurve wiedergibt, wurde versucht, die Meßpunkte der Gl. (11) anzupassen. Dabei stellte sich heraus, daß nur eine der drei Exponentialfunktionen in dem gemessenen Dosisbereich von $5 \cdot 10^{3}$ bis $5 \cdot 10^{8} \mathrm{rad}$ signifikant ist. Eine Berücksichtigung der beiden anderen Exponentialfunktionen wird erst dann möglich sein, wenn die Messungen zu niedrigen Dosiswerten hin fortgesetzt worden sind. Setzt man in Gl. (11) zur Abkürzung $h+g-f=\alpha, c=\beta$, $-h=\gamma, \sigma=\delta, D=x$, so besteht das Anpassungsproblem darin, die Parameter der Funktion

$$
\varphi(x)=\alpha+\beta x+\gamma e^{-x}
$$

im Sinne der kleinsten Quadrate so zu bestimmen, daß die Funktion

$$
S(\alpha, \beta, \gamma, \delta)=\sum_{\mathrm{i}=1}^{\mathrm{n}} p_{\mathrm{i}}\left[y_{\mathrm{i}}-\varphi\left(x_{\mathrm{i}}\right)\right]^{2}
$$

ein Minimum hat. Darin sind die $x_{\mathrm{i}}, y_{\mathrm{i}}$ die Koordinaten der Meßpunkte und $p_{\mathrm{i}}$ die zugehörigen Gewichte. Eine notwendige Bedingung für das Vorliegen eines Minimums von $S$ ist

$$
\frac{\partial S}{\partial \alpha}=\frac{\partial S}{\partial \beta}=\frac{\partial S}{\partial \gamma}=\frac{\partial S}{\partial \delta}=0 .
$$

Zur Lösung dieser Gln. wendet man üblicherweise das sogenannte gedämpfte Standardverfahren an ${ }^{20}$. In unserem Falle ist es einfacher, $\alpha, \beta$ und $\gamma$ aus den ersten drei Gl. (19) als Funktion von $\delta$ auszudrücken und in die vierte Gl. (19) einzusetzen. Das ergibt eine Gleichung

$$
F(\delta)=0 .
$$

Eine Nullstelle $\delta^{*}$ dieser Funktion ist dann zusammen mit den entsprechenden Werten $\alpha\left(\delta^{*}\right), \beta\left(\delta^{*}\right)$, $\gamma\left(\delta^{*}\right)$ ein stationärer Punkt der Funktion (18). Nach diesem Verfahren wurden die Meßpunkte von Thymin-6-T (Tab.1) jeweils mit den Gewichten $p_{\mathrm{i}}=1$ und $p_{\mathrm{i}}=1 / y_{\mathrm{i}}$ ausgeglichen, wobei die letzteren Gewichte die bessere Anpassung ergaben. Der nach Gl. (20) gefundene Wert für $\delta$ ist die einzige Nullstelle von $F$ in dem $\delta$-Intervall $\left(10^{-8}, 10^{-5}\right)$, ent-

20 D. Braess, Computing 1, 264 [1966].

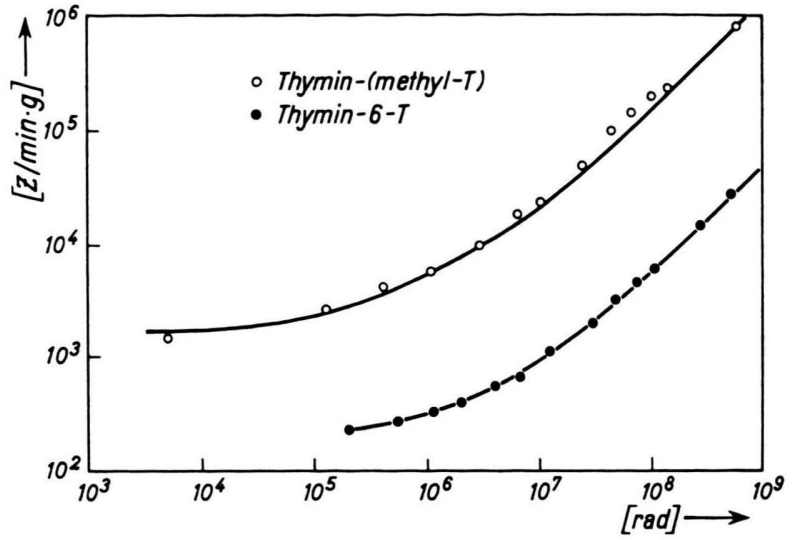

Abb. 2. Vergleich der gemessenen mit den berechneten Dosiseffektkurven für die Tritiumabspaltung aus Thymin-6-T und Thymin-(methyl-T). Die ausgezogenen Kurven sind nach den Funktionen in den Tabn. 1 und 2 berechnet.

\begin{tabular}{ccc}
\hline Dosis & $\begin{array}{c}\text { Gemessene } \\
\text { Tritiumaktivität } \\
{[Z / \mathrm{min} \cdot \mathrm{g}]}\end{array}$ & $\begin{array}{c}\text { Berechnete } \\
\text { Tritiumaktivität } \\
{[Z / \mathrm{min} \cdot \mathrm{g}]}\end{array}$ \\
\hline $2,02 \cdot 10^{5}$ & $2,20 \cdot 10^{2}$ & $2,25 \cdot 10^{2}$ \\
$5,30 \cdot 10^{5}$ & $2,70 \cdot 10^{2}$ & $2,63 \cdot 10^{2}$ \\
$1,10 \cdot 10^{6}$ & $3,20 \cdot 10^{2}$ & $3,22 \cdot 10^{2}$ \\
$1,96 \cdot 10^{6}$ & $4,10 \cdot 10^{2}$ & $3,98 \cdot 10^{2}$ \\
$4,20 \cdot 10^{6}$ & $5,40 \cdot 10^{2}$ & $5,55 \cdot 10^{2}$ \\
$6,75 \cdot 10^{6}$ & $6,70 \cdot 10^{2}$ & $7,04 \cdot 10^{2}$ \\
$1,28 \cdot 10^{7}$ & $1,11 \cdot 10^{3}$ & $1,03 \cdot 10^{3}$ \\
$3,00 \cdot 10^{7}$ & $1,98 \cdot 10^{3}$ & $1,95 \cdot 10^{3}$ \\
$4,99 \cdot 10^{7}$ & $3,10 \cdot 10^{3}$ & $3,01 \cdot 10^{3}$ \\
$7,85 \cdot 10^{7}$ & $4,56 \cdot 10^{3}$ & $4,54 \cdot 10^{3}$ \\
$1,09 \cdot 10^{8}$ & $5,80 \cdot 10^{3}$ & $6,17 \cdot 10^{3}$ \\
$2,65 \cdot 10^{8}$ & $1,42 \cdot 10^{4}$ & $1,45 \cdot 10^{4}$ \\
$5,02 \cdot 10^{8}$ & $2,77 \cdot 10^{4}$ & $2,72 \cdot 10^{4}$ \\
$\varphi(x)=3,48 \cdot 10^{2}+5,34 \cdot 10^{-5} x-1,48 \cdot 10^{2} \exp (-5,06$ \\
$\left.10^{-7} x\right)$ & & \\
\hline
\end{tabular}

Tab. 1. Gemessene und berechnete Werte zu der Dosiseffektkurve von Thymin-6-T.

\begin{tabular}{ccc}
\hline $\begin{array}{c}\text { Dosis } \\
{[\mathrm{rad}]}\end{array}$ & $\begin{array}{c}\text { Gemessene } \\
\text { Tritiumaktivität } \\
{[Z / \mathrm{min} \cdot \mathrm{g}]}\end{array}$ & $\begin{array}{c}\text { Berechnete } \\
\text { Tritiumaktivität } \\
{[Z / \mathrm{min} \cdot \mathrm{g}]}\end{array}$ \\
\hline $5,40 \cdot 10^{3}$ & $1,46 \cdot 10^{3}$ & $1,71 \cdot 10^{3}$ \\
$1,31 \cdot 10^{5}$ & $2,60 \cdot 10^{3}$ & $2,42 \cdot 10^{3}$ \\
$4,18 \cdot 10^{5}$ & $4,10 \cdot 10^{3}$ & $3,77 \cdot 10^{3}$ \\
$1,12 \cdot 10^{6}$ & $5,64 \cdot 10^{3}$ & $6,05 \cdot 10^{3}$ \\
$2,90 \cdot 10^{6}$ & $9,60 \cdot 10^{3}$ & $9,45 \cdot 10^{3}$ \\
$6,56 \cdot 10^{6}$ & $1,80 \cdot 10^{4}$ & $1,49 \cdot 10^{4}$ \\
$1,05 \cdot 10^{7}$ & $2,32 \cdot 10^{4}$ & $2,07 \cdot 10^{4}$ \\
$2,53 \cdot 10^{7}$ & $4,66 \cdot 10^{4}$ & $4,25 \cdot 10^{4}$ \\
$4,55 \cdot 10^{7}$ & $9,82 \cdot 10^{4}$ & $7,22 \cdot 10^{4}$ \\
$6,83 \cdot 10^{7}$ & $1,39 \cdot 10^{4}$ & $1,06 \cdot 10^{4}$ \\
$1,01 \cdot 10^{8}$ & $1,88 \cdot 10^{5}$ & $1,53 \cdot 10^{5}$ \\
$1,47 \cdot 10^{8}$ & $2,25 \cdot 10^{5}$ & $2,21 \cdot 10^{5}$ \\
$5,78 \cdot 10^{8}$ & $8,67 \cdot 10^{5}$ & $8,55 \cdot 10^{5}$ \\
$\psi(x)=5,28 \cdot 10^{3}+1,47 \cdot 10^{-3} x-3,60 \cdot 10^{3} \exp (-1,26 \cdot 1$, \\
$\left.10^{-6} x\right)$ & \multicolumn{3}{c}{} \\
\hline
\end{tabular}

Tab. 2. Gemessene und berechnete Werte zu der Dosiseffektkurve von Thymin-(methyl-T). 
spricht also einem absoluten Minimum der Funktion $S$ in diesem Bereich. Die so berechnete Kurve ist in Abb. 2 eingezeichnet; die gefundenen Parameter und die damit berechneten Ordinatenwerte finden sich in Tab. 1.

Die experimentellen Daten für das Thymin-(methyl-T) konnten weder mit den Gewichten $p_{\mathrm{i}}=1$ noch mit $p_{\mathrm{i}}=1 / y_{\mathrm{i}}$ auf diese Weise ausgeglichen werden. Durch Tabellieren der Funktion $F$ zeigte sich, $\mathrm{da} ß$ es in beiden Fällen keine Nullstelle der Funktion $F$ in dem $\delta$-Intervall $\left(10^{-7}, 10^{-4}\right)$ gibt. Dieses Verfahren führt hier also zu keinem Ergebnis, auch wenn man den Punkt mit der niedrigsten Dosis $\left(5,4 \cdot 10^{3} \mathrm{rad}\right)$ probeweise wegläßt.

Es wurde daher versucht, die Meßpunkte in verschiedenen Dosisbereichen getrennt anzupassen. In der Tat lassen sich die letzten acht Meßpunkte der Tab. 2 mit der Methode der kleinsten Fehlerquadrate und den Gewichten $p_{\mathrm{i}}=1$ gut durch eine Gerade $A+B x$ anpassen. Die Differenz zwischen der extrapolierten Geraden und den unteren fünf Meßwerten in Tab. 2 wurde durch eine Kurve

$$
\chi(x)=\alpha+\beta e^{-\gamma x}
$$

mit den Gewichten $p_{\mathrm{i}}=1$ nach einem ähnlichen Verfahren ${ }^{21}$ ausgeglichen. Die im gesamten Dosisbereich angepaßten Werte ergeben sich dann durch Summation der beiden erhaltenen Funktionen zu

$$
\psi(x)=A+B x-\left(\alpha+\beta e^{-\gamma x}\right) .
$$

Die Parameter und die damit angepaßten Ordinaten finden sich in Tab. 2. Abb. 2 zeigt die entsprechende Kurve. Die Bildung von $\psi(x)$ durch Subtraktion der Kurve $\chi(x)$ von der Geraden $\mathrm{A}+B x$ wird durch die Tatsache gerechtfertigt, da $\beta$ die Gerade $(A-\alpha)$ $+B x$ für die letzten acht Meßpunkte eine ebenso gute Anpassung wie $A+B x$ liefert, in dem Sinne, $\mathrm{da} ß$ in beiden Fällen die Differenzen zwischen gegebenen und angepaßten Daten dieselbe Größenordnung besitzen.

\section{Diskussion}

Aus Abb. 2 geht hervor, daß die gemessene $\mathrm{H}_{2}$ Abspaltung im Falle des Thymin-6-T sehr gut mit Hilfe unseres kinetischen Modells beschrieben werden kann. Im Falle des Thymin-(methyl-T) gelingt es nicht, bei der Anpassung der experimentellen Dosiseffektkurve ein absolutes Minimum aufzufinden.

21 H. Späтr, Commun. of the ACM 10, 87 [1967].
Möglicherweise liegt die Ursache dafür in einer Abspaltung von Methyl-T-Gruppen, die als Methan-T bzw. Äthan-T im Zählrohr eine erhöhte Tritiumwasserstoff-Aktivität vortäuschen. Das erfahrungsgemäß geringe Ausmaß dieser Abspaltung soll noch durch Bestrahlung von Thymin-(methyl $\left.-{ }^{14} \mathrm{C}\right)$ geklärt werden. Die Anpassungsfunktionen $\varphi(x)$ und $\psi(x)$ in den Tabellen 1 und 2 liefern im einzelnen folgende Konstanten der Funktion (11) :

$$
\begin{array}{ccccc} 
& \left.\begin{array}{c}
g+h-f \\
{\left[\frac{\text { Zerfälle }}{\min \cdot g}\right]}
\end{array}\right] & \left.\begin{array}{c}
c \\
\text { Zerfälle } \\
\min \cdot g
\end{array}\right] & \left.\begin{array}{c}
\sigma \\
\text { Zerfälle } \\
\min \cdot g
\end{array}\right] & {\left[\begin{array}{c}
1 \\
\text { rad }
\end{array}\right]} \\
\begin{array}{c}
\text { Thymin-6-T } \\
\text { Thymin- } \\
\text { (methyl-T) }
\end{array} & 5,28 \cdot 10^{2} & 5,34 \cdot 10^{-5} & 1,48 \cdot 10^{2} & 5,06 \cdot 10^{-7} \\
& 1,47 \cdot 10^{-3} & 3,60 \cdot 10^{3} & 1,26 \cdot 10^{-6}
\end{array}
$$

Thymin-

Aussagen über den Bereich unterhalb $5 \cdot 10^{3} \mathrm{rad}$ der Dosiseffektkurven wären mit Hilfe positionsmarkierter ${ }^{3} \mathrm{H}$-Thymine höherer spezifischer Aktivität zu gewinnen, wobei jedoch die Autoradiolyse der festen Substanzen zu Schwierigkeiten führen könnte. Erst wenn eine Anpassung der Meßwerte an die Gesamtfunktion (11) möglich ist, wird es sinnvoll sein, aus den gefundenen Parametern $\varkappa, k_{14}, \sigma, c, f, g$ und $h$ die Geschwindigkeitskonstanten der einzelnen Reaktionen zu berechnen. Die Größe $\sigma$ läßt aber bereits jetzt eine Aussage über den Dosisbereich zu, in dem die Konzentration der Thymylradikale $M$ in den Sättigungsast einmündet. Hier eröffnet sich ein interessanter Vergleich mit der von PIETsCh ${ }^{19}$ durch ESRSpektroskopie gemessenen Dosisabhängigkeit der bei der Gammabestrahlung von Thymin gebildeten Festkörperradikale, von denen eines als $\mathrm{MH}_{2}$-Radikal identifiziert ist. Subtrahiert man die bekannte $\mathrm{MH}_{2}$ Konzentration von der Gesamtspinkonzentration, so erhält man nach Pietsch eine Sättigungskurve, die durch Gl. (8) beschrieben werden kann und einen $\sigma$-Wert von $1,33 \cdot 10^{-6} \mathrm{rad}^{-1}$ besitzt. Der aus der Funktion $\psi(x)$ in Tabelle 2 gewonnene $\sigma$-Wert von $1,26 \cdot 10^{-6} \mathrm{rad}^{-1}$ steht damit in überraschender Ubereinstimmung.

Es ist bereits ausgeführt worden, daß das in dieser Arbeit diskutierte Reaktionsmodell zu einer Gleichung führt (Funktion 11), die bei positiven Konstanten $f, g$ und $h$ ebenso wie die beiden speziellen Fälle [Gl. (12) und Gl. (15) ] einen Wendepunkt besitzt. Dazu muß lediglich vorausgesetzt werden, daß entweder Reaktion 10 vernachlässigbar ist oder Reaktion 14 nicht übermäßig ins Gewicht fällt $\left(k_{14}\left[H_{2}\right]_{\mathrm{r}}<\left(k_{3}+k_{5}\right)[H]\right)$, eine Annahme, die man mit großer Wahrscheinlichkeit als gegeben ansehen kann. In diesem Fall gelingt eine Anpassung der ge- 
messenen Dosiseffektkurven an die allgemeine Funktion (11), nicht jedoch an die speziellen Funktionen (12) und (15), die unter Vernachlässigung einmal des direkten $\mathrm{H}_{2}$-Verbrauchs und zum andern der spontanen Rekombination hergeleitet wurden. Das zeigt ein Vergleich der Konstanten in den Funktionen (12) und (15) mit den Konstanten der angepaßten Funktionen in den Tabellen 1 und 2. Das absolute Glied ist in den Gl. (12) und (15) größer als der Vorfaktor der negativen Exponentialfunktionen, im Gegensatz zu den von der Anpassung gelieferten Werten; z. B. ist im Falle des Thymin-6-T $h^{\prime}-f^{\prime}=$ $348, h^{\prime}=148$ bzw. $g^{\prime}-f^{\prime \prime}=348, g^{\prime}=148$. Diese Beziehungen sind nur dann widerspruchsfrei, wenn $f^{\prime}$ bzw. $f^{\prime \prime}$ negative Größen sind. Aus Gl. (12) ist jedoch ersichtlich, daß $f^{\prime}$ niemals negativ sein kann, ohne daß auch $h^{\prime}$ negativ ist. Lediglich $f^{\prime \prime}$ kann unter bestimmten Voraussetzungen allein negativ werden, nämlich dann, wenn der $\mathrm{H}_{2}$-Verbrauch durch Reaktion 14 größer ist als die $\mathrm{H}_{2}$-Produktion durch die Reaktionen 3 und 5. (Das ist nur dann sinnvoll, wenn zusätzlich $\mathrm{H}_{2}$ durch die Reaktion 10 erzeugt wird.) In diesem Fall hätte die Funktion (15) keinen Wendepunkt. Diese Überlegungen lassen im Rahmen unseres Modells folgende Schlüsse zu:

1. Auf $\mathrm{H}_{2}$-verbrauchende Reaktionen kann nicht verzichtet werden. Um welche der beiden Reaktionen 13 oder 14 es sich dabei handelt, läßt sich vom mathematischen Standpunkt aus nicht entscheiden. $\mathrm{Ob}$ in diesen Reaktionen die Thymin- oder die $\mathrm{H}_{2}$ Moleküle als hochangeregt betrachtet werden, ist für die mathematische Analyse unerheblich. (Im letzteren Fall ist dann $\left[\mathrm{H}_{2}\right]_{\mathrm{r}}$ die Konzentration der hochangeregten $\mathrm{H}_{2}$-Moleküle.)

2. Die spontane Rekombination nach Reaktion 2 ist nur unter sehr einschränkenden und überdies wenig wahrscheinlichen Voraussetzungen entbehrlich, die erst dann geklärt werden können, wenn Meßpunkte im Dosisbereich $<10^{5} \mathrm{rad}$ vorliegen und bekannt ist, ob ein Wendepunkt auftritt oder nicht.

Im allgemeinen kann also weder die spontane Rekombination (Reaktion 2) noch der $\mathrm{H}_{2}$-Verbrauch (Reaktionen 13 und 14) vernachlässigt werden. Reaktion 10 ist dann von untergeordneter Bedeutung und dürfte als Reaktion 0. Ordnung gegenüber der Reaktion 1 nicht sehr ins Gewicht fallen. Von den anderen beiden $\mathrm{H}_{2}$-bildenden Reaktionen 3 und 5 ist eine entbehrlich. Es wurde bereits erwähnt, daß Reaktion 5 als überwiegender Donator für den molekularen Wasserstoff angesehen wird ${ }^{8}$.
Außer der $\mathrm{H}_{2}$-Konzentration ist auch die H-Konzentration in bestrahltem Thymin in Abhängigkeit von der Dosis von Interesse. Die H-Atome sind wegen ihrer geringen Konzentration einer quantitativen experimentellen Bestimmung schwer zugänglich. Hier liefert die Kinetik die Möglichkeit zur Abschätzung der H-Dosiseffektkurve. Im Rahmen des diskutierten Modells gilt Gl. (9) die H-Konzentration wieder. Im Gegensatz zur Konzentration des molekularen Wasserstoffs strebt also die Konzentration des atomaren Wasserstoffs einem Sättigungswert zu.

Trotz Übereinstimmung zwischen den Experimenten und dem kinetischen Modell muß die Frage geprüft werden, ob die vorgenommene Zuordnung eindeutig ist, d.h., ob es nicht andere modifizierte Modelle gibt, die die experimentellen Ergebnisse ebenso wiedergeben. So muß in Betracht gezogen werden, daß der molekulare Wasserstoff in Reaktionen 2. Ordnung (11 und 12) mit M- bzw. $\mathrm{MH}_{2}$-Radikalen reagieren kann, deren Konzentrationen dosisabhängig sind. Zur quantitativen Durchrechnung eines solchen Modells könnte die von Pietsch ${ }^{19}$ gemessene Dosisabhängigkeit der $\mathrm{M}$ - und $\mathrm{MH}_{2}$-Konzentrationen herangezogen werden. Aus mathematischen Gründen wurde vorerst darauf verzichtet, diese Konzentrationen hier einzubeziehen. Die Berücksichtigung der Reaktionen 11 und 12 dürfte aber im Prinzip zu keiner von Gl. (11) wesentlich verschiedenen Aussage führen, da es sich bei diesen Reaktionen ebenso wie bei der Reaktion 13 um $\mathrm{H}_{2}$-verbrauchende Reaktionen handelt, bei denen atomarer Wasserstoff entsteht. Grundsätzlich ist also ein Modell denkbar, in dem die Reaktionen 13 bzw. 14 durch die Reaktionen 11 oder 12 ersetzt sind.

Die Berücksichtigung der H-bildenden Reaktion 8 hat keinen wesentlichen modellverändernden Einfluß. Sie führt lediglich zu einer Veränderung der Größe $\varkappa$, wenn man die M-Konzentration durch Ansatz (8) ausdrückt.

Mathematisch schwieriger zu übersehen ist der Einfluß der H-verbrauchenden Reaktionen 2. Ordnung $(6,7,15)$. Falls diese gegenüber den $\mathrm{H}$-verbrauchenden Reaktionen 1. Ordnung überhaupt ins Gewicht fallen, ist denkbar, daß die $\mathrm{H}_{2}$-verbrauchenden Reaktionen gänzlich entbehrt werden können. Dann durchliefe die H-Konzentration ein Maximum, bevor sie Sättigung erreicht. Diese Frage muß solange unentschieden bleiben, bis Messungen der $\mathrm{H}_{2}$-Abspaltung auch im niedrigen Dosisbereich vorliegen. 


\section{Schlußbemerkungen}

Auf reaktionskinetischer Basis konnte unter Einschluß angeregter Moleküle ein Modell für die Wasserstoffabspaltung aus Thymin aufgestellt werden, das die experimentellen Ergebnisse wiedergibt. Die Ausdehnung der Messungen auf den niedrigen Dosisbereich $<5 \cdot 10^{3}$ rad unter Verwendung von ${ }^{3} \mathrm{H}$ Thyminen höherer spezifischer Aktivität wird Aufschluß geben über die Existenz eines Wendepunktes der Dosiseffektkurve und über die Geschwindigkeitskonstanten der an dem Modell beteiligten Reaktionen. Mit Hilfe der Wendetangente könnte die biologisch interessante Schwellenwertdosis für die Wasserstoffabspaltung ermittelt werden und aus den Geschwindigkeitskonstanten läßt sich der Anfangs- $G$ Wert $G_{0}$ (Anzahl der pro $100 \mathrm{eV}$ absorbierter Strahlungsenergie aus $1 \mathrm{~g}$ Thymin abgespaltenen $\mathrm{H}$ Atome)

$$
G_{0}=\lim _{\mathrm{D} \rightarrow 0} \frac{\mathrm{d}\left[\mathrm{H}_{2}\right] \mathrm{d}}{\mathrm{d} D}=h \sigma+g k_{14}-\varkappa f+c
$$

und die H-Dosiseffektkurve für den bei Raumtemperatur bisher nicht nachweisbaren atomaren Wasserstoff berechnen. Die diskutierten strahleninduzierten Reaktionen sind zur Deutung der Wasserstoffabspaltung aus vielen wasserstoffhaltigen organischen Verbindungen analog verwendbar. Insofern stellt das in dieser Arbeit erläuterte kinetische Modell einen Prototyp dar. Über die Anwendung des Modells auf die Kinetik der Wasserstoffabspaltung aus Uracil wird später berichtet werden.
Wir danken Herrn Prof. Dr. W. Seelmann-EgGebert (Institut für Radiochemie, Kernforschungszentrum Karlsruhe) und Herrn Prof. Dr. A. Catsch (Institut für Strahlenbiologie, Kernforschungszentrum Karlsruhe) für ihr Interesse an dieser Arbeit.

\section{Anhang}

Läßt man in dem vorgeschlagenen Modell die spontanen Rekombinationen 2 und 3 außer acht und berücksichtigt als $\mathrm{H}_{2}$-verbrauchende Reaktionen sowohl 13 als auch 14, so ergeben sich an Stelle der Gln. (3) bis (6) für die Konzentrationen des atomaren und des molekularen Wasserstoffs folgende Differentialgleichungen:

$$
\begin{aligned}
& \frac{\mathrm{d}[H]}{\mathrm{d} D}=k_{1}-k_{4}[H]-k_{5}[H]+k_{13}\left[H_{2}\right]_{\mathrm{r}} \\
& \frac{\mathrm{d}\left[H_{2}\right]}{\mathrm{d} D}=k_{10}+k_{5}[H]-\left(k_{13}+k_{14}\right)\left[H_{2}\right]_{\mathrm{r}} \\
& \frac{\mathrm{d}\left[H_{2}\right]_{\mathrm{r}}}{\mathrm{d} D}=k_{10 \mathrm{r}}+k_{5 \mathrm{r}}[H]-\left(k_{13}+k_{14}\right)\left[H_{2}\right]_{\mathrm{r}} \\
& \frac{\mathrm{d}\left[H_{2}\right]_{\mathrm{d}}}{\mathrm{d} D}=k_{10 \mathrm{~d}}+k_{5 \mathrm{~d}}[H] .
\end{aligned}
$$

Setzt man die aus Gl. (A 3) berechnete H-Konzentration

$$
[H]=\frac{1}{k_{5 \mathrm{r}}}\left[\frac{\mathrm{d}\left[H_{2}\right]_{\mathrm{r}}}{\mathrm{d} D}+\left(k_{13}+k_{14}\right)\left[H_{2}\right]_{\mathrm{r}}-k_{10 \mathrm{r}}\right]
$$

in Gl. (A 1) ein, so ergibt sich eine inhomogene lineare Differentialgleichung 2. Ordnung für die $H_{2 r^{-}}$ Konzentration:

$$
\frac{\mathrm{d}^{2}\left[H_{2}\right]_{\mathrm{r}}}{\mathrm{d} D^{2}}+\frac{\mathrm{d}\left[H_{2}\right]_{\mathrm{r}}}{\mathrm{d} D}\left(k_{4}+k_{5}+k_{13}+k_{14}\right)+\left[H_{2}\right]_{\mathrm{r}}\left[\left(k_{4}+k_{5}\right)\left(k_{13}+k_{14}\right)-k_{5 \mathrm{r}} k_{13}\right]-k_{1} k_{5 \mathrm{r}}-k_{10 \mathrm{r}}\left(k_{4}+k_{5}\right)=0 .
$$

Mit den Anfangsbedingungen, daß die Konzentrationen des atomaren und des molekularen Wasserstoffs zu Beginn der Bestrahlung gleich Null sind $\left([H]=\left[H_{2}\right]_{\mathrm{r}}=0\right.$ für $\left.D=0\right)$, hat die Differential-

gleichung (A 6) die Lösung:

$$
\left[H_{2}\right]_{\mathrm{r}}=-p[1-\exp (a D)]+q[1-\exp (-b D)] \text {. }
$$

Darin sind

$$
\begin{aligned}
& a=\frac{k_{4}+k_{5}+k_{13}+k_{14}}{2}+\sqrt{\left(\frac{k_{4}+k_{5}+k_{13}+k_{14}}{2}\right)^{2}-\left(k_{4}+k_{5}\right)\left(k_{13}+k_{14}\right)+k_{13} k_{5 \mathrm{r}},} \\
& b=\frac{k_{4}+k_{5}+k_{13}+k_{14}}{2}-\sqrt{\left(\frac{k_{4}+k_{5}+k_{13}+k_{14}}{2}\right)^{2}-\left(k_{4}+k_{5}\right)\left(k_{13}+k_{14}\right)+k_{13} k_{5 \mathrm{r}},} \\
& p=\frac{k_{1} k_{5 \mathrm{r}}+k_{10 \mathrm{r}}\left(k_{4}+k_{5}-a\right)}{a^{2}-a b} \quad \text { und } \\
& q=\frac{k_{1} k_{5 \mathrm{r}}+k_{10 \mathrm{r}}\left(k_{4}+k_{5}-b\right)}{a b-b^{2}} .
\end{aligned}
$$


Durch Einsetzen dieser Lösung in Gl. (A 5) ergibt sich die Konzentration des atomaren Wasserstoffs zu

$$
[H]=\frac{p}{k_{5 \mathrm{r}}}\left[a-\left(k_{13}+k_{14}\right][1-\exp (-a D)]-\frac{q}{k_{5 \mathrm{r}}}\left[b-\left(k_{13}+k_{14}\right)\right][1-\exp (-b D)] .\right.
$$

Nunmehr ist man in der Lage, Gl. (A 4) mit der Anfangsbedingung $\left[H_{2}\right]_{\mathrm{d}}=0$ für $\mathrm{D}=0$ zu integrieren und erhält

mit

$$
\begin{gathered}
{\left[H_{2}\right]_{\mathrm{d}}=-\frac{p k_{5 \mathrm{~d}}}{a k_{5 \mathrm{r}}}\left[a-\left(k_{13}+k_{14}\right)\right][1-\exp (-a D)]+\frac{q k_{5 \mathrm{~d}}}{b k_{5 \mathrm{r}}}\left[b-\left(k_{13}+k_{14}\right)\right][1-\exp (-b D)]+c D} \\
c=\frac{k_{13}\left(k_{5 \mathrm{~d}} k_{10 \mathrm{r}}-k_{10 \mathrm{~d}} k_{5 \mathrm{r}}\right)+\left(k_{13}+k_{14}\right)\left[k_{1} k_{5 \mathrm{~d}}+k_{10 \mathrm{~d}}\left(k_{4}+k_{5}\right)\right]}{\left(k_{4}+k_{5}\right)\left(k_{13}+k_{14}\right)-k_{13} k_{5 \mathrm{r}}}
\end{gathered}
$$

Wegen der Beziehung (2) gibt Gl. (A 9) bei relativ hohen Dosen bereits die gemessene Gesamtwasserstoffkonzentration $\left[\mathrm{H}_{2}\right]$ wieder. Im Bereich kleiner Dosiswerte muß auch noch $\left[\mathrm{H}_{2}\right]_{\mathrm{r}}$ berücksichtigt werden. Nach Gl. (1) führt die Addition der Gln. (A 7) und (A 9) zur gesamten $\mathrm{H}_{2}$-Konzentration

$$
\begin{gathered}
{\left[H_{2}\right]=-f[1-\exp (-a D)] \quad(\mathrm{A} 10)} \\
+g[1-\exp (-b D)]+c D,
\end{gathered}
$$

wobei

$$
f=\frac{k_{1} k_{5 \mathrm{r}}+k_{10 \mathrm{r}}\left(k_{4}+k_{5}-a\right)}{a^{2}-a b}\left(\frac{k_{5 \mathrm{~d}}}{k_{5 \mathrm{r}}} \frac{a-\left(k_{13}+k_{14}\right)}{a}+1\right)
$$

und

$$
g=\frac{k_{1} k_{5 \mathrm{r}}+k_{10 \mathrm{r}}\left(k_{4}+k_{5}-b\right)}{a b-b^{2}}\left(\frac{k_{5 \mathrm{~d}}}{k_{5 \mathrm{r}}} \frac{b-\left(k_{13}+k_{14}\right)}{b}+1\right)
$$

